\title{
Acknowledgment to Reviewers of Non-Coding RNA in 2020
}

\author{
Non-Coding RNA Editorial Office
}

MDPI AG, St. Alban-Anlage 66, 4052 Basel, Switzerland

Citation: Non-Coding RNA

Editorial Office. Acknowledgment to

Reviewers of Non-Coding RNA in

2020. Non-Coding RNA 2021, 7, 7.

https://doi.org/10.3390/ncrna7010007

Published: 25 January 2021

Publisher's Note: MDPI stays neutral with regard to jurisdictional claims in published maps and institutional affiliations.

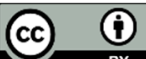

Copyright: (c) 2021 by the authors. Submitted for possible open access publication under the terms and conditions of the Creative Commons Attribution (CC BY) license (http://creativecommons.org/licenses/by/4.0/).

Peer review is the driving force of journal development, and reviewers are gatekeepers who ensure that Non-Coding RNA maintains its standards for the high quality of its published papers. Thanks to the cooperation of our reviewers, in 2020, the median time to first decision was 17 days and the median time to publication was 39.5 days. The editors would like to express their sincere gratitude to the following reviewers for their precious time and dedication, regardless of whether the papers were finally published:

Adriaens, Carmen

Agnelli, Luca

Ali, Ali

Allegrucci, Cinzia

Allmer, Jens

Anastasiadou, Eleni

Andreassen, Rune J.

Andrés-León, Eduardo

Bajan, Sarah

Balatti, Veronica

Biagioli, Marta

Biamonti, Giuseppe

Bilia, Anna Rita

Blandino, Giovanni

Bracken, Cameron

Branlant, Christiane

Bronisz, Agnieszka

Brown, Jessica A.

Calabrese, J. Mauro

Carmichael, Gordon G.

Cerase, Andrea

Chakrabarti, Kausik

Chang, Suhwan

Charchar, Fadi

Chen, Ling-Ling

Cheng, Haizi

Chiu, Hua-Sheng

Chiu, Norman

Chowdhury, Imran Hussain

Clark, Brian

Clezardin, Philippe

Conigliaro, Alice

Cook, Atlanta G.

Coulouarn, Cédric

Dajas-Bailador, Federico
De Lencastre, Alex

Deryusheva, Svetlana

Di Leva, Gianpiero

Ding, Yiliang

Djuranović, Sergej

Do, Duy Ngoc

Doody, Gina

Dundr, Miroslav

Enguita, Francisco J.

Eslam, Mohammed

Fabbri, Muller

Falzone, Luca

Fatkhudinov, Timur Kh.

Floris, Matteo

García-Ordás, María Teresa

Gaviraghi, Marco

Gerber, André

González-Vallinas, Margarita

Göringer, H. Ulrich

Gorospe, Myriam

Gradia, Daniela

Guang, Shouhong

Gururajan, Anand

Happel, Christine M.

Haspel, Nurit

Hendig, Doris

Henriksson, Johan

Hewitson, James P.

Higgs, Paul

Hueso, Miguel

Hutchinson, John N.

Idogawa, Masashi

Johnson, Aaron

Johnson, Rory

Kang, Yang Jae 
Kawakami, Junji

Klec, Christiane

Klinge, Carolyn M.

Köhn, Marcel

Krasikova, Alla

Krause, Henry M.

Kristensen, Lasse S.

Le Grice, Stuart F. J.

Lee, Yong Sun

Leisegang, Matthias S.

Leucci, Eleonora

Li, Haobo

Li, Howard

Liang, Tingming

Lingner, Joachim

LoVerde, Phil

Makalowska, Izabela

Marciniak, Katarzyna

Martinez, Ernest

Mayer, Bryan T.

Meister, Gunter

Metere, Alessio

Militello, Giuseppe

Montes Resano, Marta

Morgan, Marcos

Morris, Kevin

Mukhopadhyay, Arijit K.

Muxel, Sandra Marcia

Nakagawa, Shinichi

Nigita, Giovanni

Nociti, Viviana

Ogami, Koichi

Ozer, Hatice Gulcin
Parchem, Ronald J.

Pasini, Luigi

Payne, Annette

Prasanth, Kannanganattu V.

Rasin, Mladen Roko

Raudsepp, Terje

Rayner, Simon

Renwick, Neil

Rodilla, Verónica

Romani, Andrea

Roy, Bhaskar

Sablina, Anna

Schaefer, Matthias

Sehgal, Lalit

Shelkovnikova, Tatyana A.

Shiina, Marisa

Shyu, Ann-Bin

Smyth, Redmond

Stafforst, Thorsten

Stathopoulos, Constantinos

Sztuba-Solinska, Joanna

Taguchi, Yoshihiro

Tonge, Daniel

Torres, Adrian Gabriel

Uchida, Shizuka

Valadkhan, Saba

Waghmare, Sakharam

Wellinger, Raymund J.

Wery, Maxime

Williams, Joanna

Yan, Wei

Yukawa, Yasushi

Żok, Tomasz 